\title{
Status social subjetivo em modalidades esportivas coletivas
}

\author{
Subjective social status in collective sports \\ Estado social subjetivo en deportes colectivos
}

Recebido: 16/01/2022 | Revisado: 20/01/2022 | Aceito: 25/01/2022 | Publicado: 27/01/2022

ORCID: https://orcid.org/0000-0001-9346-1460 Universidade Católica de Brasília, Brasil E-mail: elisapinheiroferrari@gmail.com

Silvana Carolina Fürstenau dos Santos ORCID: https://orcid.org/0000-0003-0629-4313 Universidade Católica de Brasília, Brasil E-mail: silvanafurstenau@gmail.com

Leyla Régis de Meneses Carvalho Sousa Carvalho ORCID: https://orcid.org/0000-0001-9912-4166 Universidade Católica de Brasília, Brasil E-mail: leyla.regis@hotmail.com

Diego Soares Souza

ORCID: https://orcid.org/0000-0002-0705-3792 Universidade Católica de Brasília, Brasil E-mail: dinho_souza@hotmail.com

Julianna Mendes de Matos Souza ORCID: https://orcid.org/0000-0002-5463-6990 Universidade Católica de Brasília, Brasil E-mail: juli_mmatos@hotmail.com

Maria Bonfim Carlos de Paulo ORCID: https://orcid.org/0000-0003-2302-338X Universidade Católica de Brasília, Brasil E-mail: mariabonfimcsilva@ hotmail.com Fernando Luiz Cardoso ORCID: https://orcid.org/0000-0002-3074-0988 Universidade do Estado de Santa Catarina, Brasil E-mail: fernandocardoso.ph.d.lagesc@ gmail.com

\begin{abstract}
Resumo
O status social subjetivo é a percepção de um indivíduo acerca da sua posição social nos mais diversos contextos de vida. O objetivo deste estudo foi comparar o status social subjetivo entre atletas de modalidade esportivas coletivas. Participaram deste estudo 115 atletas do sexo masculino das seguintes modalidades: futebol, voleibol, handebol e basquetebol. A avaliação da percepção do status social subjetivo foi realizada por meio da escala MacArthur. Para analise dos dados foram utilizados os testes Qui-quadrado, Anova one way e Kolmogorov Smirnov. De acordo com os dados, pode-se observar que os atletas de futebol foram os que apresentaram os menores escores de status social subjetivo atual no clube $(\mathrm{X}: 4.9 ; \pm 2.0)$ e no contexto familiar $(\mathrm{X}: 7.0 ; \pm 2.0)$. Além disso, os atletas de futebol apresentam maiores prevalências de insatisfação $(87.2 \%$; $n=42)$. Concluí-se que os atletas de futebol têm uma maior percepção de insatisfação com o status social subjetivo.
\end{abstract}

Palavras-chave: Atletas; Impacto psicossocial; Hierarquia social.

\begin{abstract}
Subjective social status is the perception of an individual about his social position in the most diverse contexts of life. The aim of this study was to compare the subjective social status among collective sport athletes. A total of 115 male athletes participated in this study: soccer, volleyball, handball and basketball. The assessment of the perception of subjective social status was performed using the MacArthur scale. For data analysis the chi-square, Anova one way and Kolmogorov Smirnov tests were used. According to the data, it can be observed that the soccer athletes presented the lowest scores of current subjective social status in the club (X: $4.9 ; \pm 2.0)$ and in the family context (X: $7.0 ; \pm 2.0)$. In addition, soccer athletes have a higher prevalence of dissatisfaction $(87.2 \% ; n=42)$. It was concluded that soccer athletes have a higher perception of dissatisfaction with subjective social status.
\end{abstract}

Keywords: Athletess; Psychosocial impact; Social hierarchy.

\section{Resumen}

El estatus social subjetivo se refiere a la percepción de un individuo de su posición social en los contextos más diversos de la vida. El objetivo de este estudio fue comparar el estado social subjetivo entre los atletas de deportes 
colectivos. Un total de 115 atletas masculinos participaron en este estudio: fútbol, voleibol, balonmano y baloncesto. La percepción del estado social subjetivo se evaluó utilizando la escala de Status Social Subjetivo de MacArthur. La evaluación de la percepción del estado social subjetivo se realizó mediante la escala de MacArthur. Para el análisis de los datos, se utilizaron las pruebas de chi-cuadrado, Anova one way y Kolmogorov Smirnov. De acuerdo con los datos, se puede observar que los atletas de fútbol presentaron las puntuaciones más bajas del estado social subjetivo actual en el club (X: $4.9 ; \pm 2.0)$ y en el contexto familiar (X: $7.0 ; \pm 2.0)$. Además, los atletas de fútbol tienen una mayor prevalencia de insatisfacción $(87.2 \%$; $=42)$. Se concluye que los atletas de fútbol tienen una mayor percepción de insatisfacción con el estatus social subjetivo.

Palabras clave: Atletas; Impacto psicosocial; Jerarquía social.

\section{Introdução}

Atualmente tem-se verificado um aumento no número de pesquisas voltadas para a compreensão das variáveis psicológicas e sociais inerentes as práticas esportivas (Bertoldi et al., 2018; Bonfim et al., 2018). Dentre elas, destaca-se o status social subjetivo (SSS), uma escala analógica de representação de uma hierarquia qualitativa do melhor para o pior considerando os contextos, social, familiar e profissional (Silva et al., 2018).

Os indicadores sociais se subdividem em objetivos e subjetivos. Nesse contexto, é possível observar que a renda e a classe social são os indicadores mais utilizados pelos estudos, as quais são classificadas como objetivos. No que tange aos subjetivos, estes podem ser definidos como sendo a constituição dos fatores intrínsecos e extrínsecos inerentes a cada sujeito. Dessa maneira, esses indicadores têm um importante papel para a compreensão da posição social percebida pelos sujeitos no contexto social ao qual pertencem (Freitas et al., 2016; Santos et al., 2018).

De acordo com Euteneuer (2014) a percepção do SSS é uma medida que abrange a posição social do sujeito em determinados ambientes (família, comunidade, trabalho, escola), e desempenha um importante papel na compreensão da posição social percebida e idealizada dos mesmos na sociedade.

No âmbito esportivo, em especial nas modalidades de alto rendimento, as cobranças emergem de múltiplas direções, como por exemplo, do técnico, da torcida, da família e da sociedade em geral, os quais influenciam, efetivamente, sobre os atletas na construção da percepção do status social subjetivo (Hoshino et al., 2007; Loughead et al., 2006). Dentre os esportes coletivos, de acordo com a literatura, o futebol de campo e o futsal têm maior destaque entre a população em geral, pois são esportes que se constituem como importante elemento da cultura nacional e, portanto, têm em sua essência a função de transformar e socializar. Além disso, existe a crença popular de que todo brasileiro já nasce com o potencial de jogador, pois estamos no país do futebol, fato que influencia os meninos a percorrerem o sonho de ser um jogador profissional, a fim de ascender financeiramente e publicamente (Rinaldi, 2000; Tedesco, 2014; Zaratim, 2012).

A procura pela profissionalização entre os atletas amadores de futebol é intensa, motivados pela fama, dinheiro e status de ídolos (Morato et al., 2011) geralmente influenciados pela família, mídia e pelos anônimos do futebol (Anjos et al., 2012) o que gera baixos níveis de status sociais entre os atletas amadores e principalmente reservas (Silva et al., 2018).

Medeiros, Ferrari e Cardoso (2014), ao avaliar o SSS de atletas de futebol em formação identificaram uma média 4,5 pontos na percepção atual do seu status no clube, desejando escores acima de 9,0 pontos, o que demonstra que os atletas estão insatisfeitos com seu status, e que pode ser justificado pelo fato das referências serem provenientes de atletas famosos e estruturados no cenário do futebol, os quais apresentam uma estabilidade financeira (Silva et al., 2018). A comparação entre as modalidades esportivas envolvendo atletas de futsal e futebol foi evidenciada no estudo de Silva et al., (2018), cujos resultados mostram que os jogadores de futebol apresentam baixos valores de SSS no âmbito do clube (ambiente), quando comparados aos atletas de futsal. 
A partir do contexto apresentado, o objetivo do presente estudo foi comparar o SSS de atletas de modalidades coletivas, futebol, voleibol, handebol e basquetebol. Hipotetizando que os atletas de futebol apresentaram status atual no clube e na família inferiores em relação aos demais atletas.

\section{Metodologia}

\subsection{Tipo de estudo}

Trata-se de um estudo transversal, delineamento caracterizado pela não intervenção dos autores nos fenômenos sob estudo, que se limitam a observar e a coletar, de maneira sistemática, os dados obtidos (Zangilorami-Raimundo, Echeimberg \& Leone, 2018). Este estudo faz parte de um estudo maior, intitulado "Identidade Esportiva e Artística de Atletas e Dançarinos". Aprovado pelo Comitê de Ética para Pesquisa (CEP) com Seres Humanos da Universidade do Estado de Santa Catarina (UDESC), sob o protocolo $\mathrm{n}^{\circ} 275381 / 2013$.

\subsection{Amostra}

A população foi composta por atletas de Santa Catarina, praticantes de futebol, futsal, voleibol, handebol e basquete. A amostra não probabilística foi intencionalmente composta por atletas da mesorregião do oeste de Santa Catarina, de acordo com os seguintes critérios de inclusão: ter idade mínima de 16 anos, ser federado por clube, associação ou departamento esportivo por pelo menos um ano, ter treinado sistematicamente por pelo menos 1 ano, com uma frequência de 3 vezes semanal, e estar treinando regularmente durante o período de coleta de dados.

\subsection{Instrumentos e procedimentos de coleta de dados}

O SSS foi avaliado por meio da aplicação da Escala MacArthur de Versão de SSS para Jovens. Esse instrumento é retratado por uma "escada social" (Figura 1) representando a escola, onde as pessoas com as notas mais altas, maior respeito e maior posição social estão no topo ou no degrau mais alto (etapa 10). Já as pessoas que têm as menores pontuações, sem respeito, ninguém quer ficar perto e ter baixo status social estão no degrau mais baixo (passo 1ou etapa 1). Para este estudo a escala foi adaptada para o ambiente esportivo, tendo os atletas revelado as autopercepções de suas posições no clube e as posições em que desejavam figurar, além de suas posições no contexto familiar.

Figura 1. MacArthur Versão para Escala de SSS para Jovens.

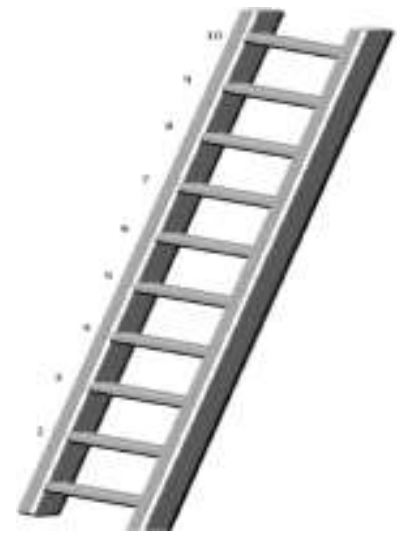

Fonte: Goodman et al., (2001). 
O indivíduo deve marcar um "X” e o número (passo) correspondente à sua autopercepção. Para o contexto brasileiro, esta ferramenta não foi validada. No entanto, Goodman et al. (2001) encontraram valores de coeficiente de correlação intraclasse de 0.73 para o contexto social e 0.79 para o familiar, indicando excelente confiabilidade para a população em que foi desenvolvido. A variável satisfação com o SSS foi obtida mediante a subtração do valor obtido com o status atual pelo idealizado, sendo que os indivíduos com resultados iguais a zero foram classificados como "satisfeitos"; valores positivos "insatisfeito por alto status" e negativos "insatisfeito por baixo status". conforme proposto e utilizado por Medeiros et al. (2014) e Freitas et al. (2016).

A modalidade esportiva foi identificada pelos pesquisadores que realizaram as entrevistas com os técnicos, a fim de identificar quais atletas atendiam aos critérios de inclusão.

As coletas de dados foram realizadas durante os jogos abertos de Santa Catarina por pesquisadores previamente treinados.

\subsection{Análise de dados}

Para análise de dados, inicialmente foi realizado o teste de Kolmogorov Smirnov para verificar a distribuição dos dados. Como os dados apresentaram distribuição normal, a comparação dos escores do SSS atual e ideal no clube e contexto familiar nas modalidades esportivas (futebol, futsal, voleibol, handebol e basquetebol) avaliadas foi verificada por meio do teste Anova one way. O teste de Qui-quadrado e Exato de Fisher foi utilizado para testar a associação entre o SSS e as modalidades esportivas (futebol, futsal, voleibol, handebol e basquetebol).

O nível de confiança foi estabelecido em 5\%. As análises foram realizadas no programa estatístico Statistical Package for the Social Sciences (SPSS) ${ }^{\circledR}$, versão 20.0.

\section{Resultados e Discussão}

Foram avaliados 115 atletas com média de idade de 19.1 ( \pm 4.7) anos, distribuídos nas seguintes modalidades esportivas, futebol $(n=47)$; futsal $(n=28)$; voleibol $(n=16)$; handebol $(n=14)$; basquetebol $(n=10)$.

Ao comparar os escores obtidos com a satisfação com o SSS atual e ideal no clube e no contexto familiar entre as modalidades esportivas coletivas investigadas, observou-se diferença somente para os atletas de futebol (p-valor $\leq 0.05$ ) com relação a satisfação com o SSS atual no clube e na família, sendo os valores destes inferiores aos relatados pelos atletas das outras modalidades (Tabela 1).

Tabela 1. Comparação dos escores de satisfação com o SSS atual e ideal no clube e no ambiente familiar de acordo com a modalidade esportiva atletas federados do oeste Catarinense.

\begin{tabular}{|c|c|c|c|c|c|}
\hline \multicolumn{6}{|c|}{ Modalidades Esportivas } \\
\hline $\begin{array}{l}\text { Satisfação com o } \\
\text { SSS }\end{array}$ & $\begin{array}{c}\text { Futebol } \\
\text { Média (DP) }\end{array}$ & $\begin{array}{c}\text { Futsal } \\
\text { Média (DP) }\end{array}$ & $\begin{array}{c}\text { Basquete } \\
\text { Média (DP) }\end{array}$ & $\begin{array}{c}\text { Handebol } \\
\text { Média (DP) }\end{array}$ & $\begin{array}{c}\text { Voleibol } \\
\text { Média (DP) }\end{array}$ \\
\hline Atual no clube & $4.9(2.0)^{\mathrm{a}}$ & $6.7(2.2)^{\mathrm{b}}$ & $7.1(1.0)^{\mathrm{b}}$ & $6.5(2.0)^{\mathrm{b}}$ & $7.0(1.8)^{\mathrm{b}}$ \\
\hline Ideal no clube & $9.5(1.0)^{\mathrm{a}}$ & $9.1(1.6)^{\mathrm{a}}$ & $8.9(1.1)^{\mathrm{a}}$ & $9.4(0.9)^{\mathrm{a}}$ & $9.4(0.9)^{\mathrm{a}}$ \\
\hline Família & $7.0(2.0)^{\mathrm{a}}$ & $8.4(1.7)^{b}$ & $8.4(1.6)^{\mathrm{b}}$ & $8.5(1.1)^{b}$ & $7.8(1.8)^{\mathrm{b}}$ \\
\hline
\end{tabular}

SSS: Status Social Subjetivo; DP: Desvio-Padrão. ${ }^{a}$ Anova one way. Letras iguais (a; b) indicam similaridade de médias nas comparações das variáveis do SSS (p-valor > 0.05) e letras diferentes indicam diferenças estatisticamente significantes nas médias das varáveis do SSS entre as modalidades esportivas ( $\mathrm{p}$-valor $\leq 0.05$ ). Fonte: Autores $(2021)$. 
Na Tabela 2 é apresentada a associação entre satisfação com o SSS e as modalidades esportivas. De forma geral, de acordo com os dados apresentados na tabela pode-se verificar que independente da modalidade, todos os atletas investigados apresentaram altas prevalências de insatisfação por baixo status, sendo os de futebol, os mais insatisfeitos (p-valor $\leq 0.05$ ).

Tabela 2. Associação entre modalidade e satisfação com o SSS entre atletas federados do oeste Catarinense.

\begin{tabular}{ccccc}
\hline Modalidade & $\begin{array}{c}\text { Satisfeito } \\
\boldsymbol{\%}(\mathbf{n})\end{array}$ & $\begin{array}{c}\text { Insatisfeito por alto } \\
\boldsymbol{\%}(\mathbf{n})\end{array}$ & $\begin{array}{c}\text { Insatisfeito por baixo } \\
\boldsymbol{\%}(\mathbf{n})\end{array}$ & p-valor \\
\hline Futebol & $6.4(03)$ & $6.4(03)$ & $87.2(41)$ & \\
Futsal & $32.1(09)$ & $21.4(06)$ & $46.4(13)$ & 0.02 \\
& & & & \\
Voleibol & $12.5(02)$ & $18.8(03)$ & $68.2(11)$ & \\
Handebol & $28.4(04)$ & $7.1(01)$ & $64.3(09)$ & \\
Basquetebol & $0.0(00)$ & $50.0(05)$ & $50.0(05)$ & \\
\hline
\end{tabular}

\%: frequência relativa; n: frequência absoluta. Fonte: Autores (2021).

\subsection{Discussão}

O status social como constructo é composto por diversos atributos, e sua interpretação é resultado de uma avaliação realizada pelas pessoas, associado à superioridade e inferioridade, identificado como sinônimo de prestígio, ou ainda tratado por uma escala de avaliação dos recursos socioeconômicos; e, algumas vezes, indicado por meio de status jurídicos, como o estado civil (Freitas et al. 2016; Silva et al. 2018).

Nessa perspectiva, o status se apresenta como uma característica fundamental e presente em todas as relações sociais e refere-se a posições e papéis atribuídos as pessoas em cada grupo social (Magee \& Galinski, 2009). No meio esportivo, a estimativa do SSS se caracteriza como uma fonte de identificação coletiva, principalmente no futebol, compreendido como um fenômeno de socialização e transmissão de valores sociais (Medeiros et al. 2014).

No presente estudo pode-se observar que os atletas de futebol atribuiram valores menores para o seu status em relação aos seus pares das outras modalidades esportivas investigadas, tanto para a autopercepção do SSS real no clube quanto no contexto familiar.

A diferença encontrada pode ser explicada pela ampla disseminação da prática do futebol no país. A popularidade desse esporte, aliada a ascensão social e econômica, faz com que os jogadores das categorias de base e, principalmente, os seus familiares, invistam precocemente nessa profissão, já que passou a ser uma possibilidade, através do esporte, de se ter um futuro promisor (Morato, et al. 2011; Rial, 2008; Tedesco, 2014)

Porém, nem todos os atletas conseguem alcançar a tal almejada ascensão profissional. Consequentemente, o salário da maioria dos que escolhem esta profissão não atende ao desejo de mobilidade social dos atletas, gerando um desencanto e causando, assim, um baixo status social (Morato et al. 2011).

Além disso, no contexto de seus clubes, os futebolistas não se percebem tão importantes e fundamentais como gostariam, ainda mais se comparados aos praticantes das outras modalidades esportivas. O futebol, por tudo o que já foi 
mencionado, é caracterizado por apresentar um ambiente formativo mais competitivo. Isso significa que os atletas são expostos, ainda muito jovens, a um contexto de pressões e cobranças em seus clubes (Tedesco; 2014; Zaratim, 2012).

O presente estudo apresenta limitações como a utilização de um instrumento não validado para a população brasileira. Trata-se da escala de McArthur, a qual não é utizada para a população jovem e para o contexto esportivo, mas inexistindo uma escala para esse fim específico, esta se mostrou apropriada. Além disso, a natureza transversal da pesquisa não permite estabelecer relações de causa e efeito. Portanto, embora a presente pesquisa acrescente informações à literatura nessa área, novos estudos são recomendados para compreender as consequências advindas da pressão psíquica e social sobre o desempenho dos atletas (Duarte, Teques, \& Silva, 2017; Freitas et al. 2016).

\section{Considerações Finais}

Com base nos resultados do presente estudo, conclui-se que os atletas de futebol do universo investigado atribuíram um menor SSS quando comparados com os atletas das outras modalidades investigadas. Além de apresentar maior insatisfação com status social subjetivo quando comparados a atletas das outras modalidades, apesar destes também estarem insatisfeitos com os seus status.

A realização de novas pesquisas é requerida a fim de confirmar os achados e identificar as variáveis associadas a este desfecho. Sugerimos que as próximas pesquisas sobre o assunto se dediquem a analisar também o universo das modalidades esportivas coletivas femininas, de forma a expandir a discussão para a problemática da relação entre gênero e esporte, com a análise de dados acerca do SSS de mulheres envolvidas nessas práticas esportivas e eventuais comparações entre as experiências masculinas e femininas.

\section{Referências}

Anjos, J. L., Saneto, J. G. \& Oliveira, A. A. (2012). Futebol, imagens e profissionalizacão: a bola rola nos sonhos dos adolescentes. Movimento, 18(1),125-47.

Bertoldi, R., Bandeira, P. F. R., Barbosa, M. L. L., Costa, J. L. da. \& Balbinotti, M. A. A. (2018). Evidências de validade do inventário de sinais e dintomas de Burnout para atletas. In Resumos de Comunicações Científicas, XVII Congresso Brasileiro e X Congresso Internacional de Psicologia do Esporte e do Exercício-CONBIPE.

Balbinotti, C. A. A. (2019). Fatores preditores dos sinais e sintomas do Burnout em atletas brasileiros profissionais de futsal. Revista Brasileira de Psicologia do Esporte, 9, (2),131-144.

Bonfim, G. M. A. do. \& Meira Junior, C. M. (2018). Traços de personalidade de nadadores de velocidade, meio-fundo e fundo. Revista Brasileira de Psicologia do Esporte, 8(2), 52-63.

Duarte, D., Teques, P. \& Silva, C. (2017). Liderança e satisfação no futebol: testagem da congruência com recurso à análise de equações estruturais. Revista Iberoamericana de Psicología del Ejercicio y el Deporte, 12(1), 71-82.

Euteneuer, F. (2014). Subjective social status and health. Current Opinion in Psychiatry, 27(5), 337-43.

Freitas, K. D., Ferrari, E. P., Vieira, M. P., da Silva, W. R., de Carvalho, H. P. \& Cardoso, F. L. (2016). Association of subjective social status and sociodemographic indicators in athletes. Revista Brasileira de Cineantropometria e Desempenho Humano. 18(5):591-601.

Goodman, E., Adler, N., Kawachi, I., Frazier, L., Huang, B. \& Colditz, G. A. (2001). Adolescents' perceptions of social status: development and evaluation of a new indicator. Pediatrics;108(2): E31.

Hoshino, E. F., Sonoo, C. N. \& Vieira, L. F. (2007). Perfil de liderança: uma análise no contexto esportivo de treinamento e competição. Revista da Educação Física, 18(1), 77-83.

Loughead, T. M., Hardy, J. \& EYS, M. A. (2006). The nature of athlete leadership. Journal of Sport Behavior, $29(2), 142$.

Magee, J. C. \& Galinski, A. D. (2009). Social Hierarchy: The Self-Reinforcing Nature of Power and Status. The Academy of Management Annals, 2(1), 351398.

Medeiros, T. E., Ferrari, E. P. \& Cardoso, F. L. (2014). Relação entre status social subjetivo e esquemas de gênero do autoconceito em jogadores de futebol. Pesquisa prática psicossociais; 9(1):106-117.

Morato, M. P., Giglio, S. S. \& Gomes, M. S. P. (2011). A construção do ídolo no fenômeno futebol. Motriz, 17(1), 1-10. 
Research, Society and Development, v. 11, n. 2, e33911225806, 2022

(CC BY 4.0) | ISSN 2525-3409 | DOI: http://dx.doi.org/10.33448/rsd-v11i2.25806

Rial, C. (2008). Rodar: a circulação dos jogadores de futebol brasileiros no exterior. Horizontes Antropológicos, 14(30), 21-65.

Rinaldi, W. (2000). Futebol: manifestação cultural e ideologização. Revista de Educação Física da UEM; 11, $167-72$.

Santos, M. O., Barbosa, D. G., Rebelatto, C. F., Beltrame, T. S. \& Felden, E. P. G. (2018). Status social subjetivo na escola e nas aulas de educação física. Revista Brasileira de Ciências do Esporte, 40(1), 77-86. https://dx.doi.org/10.1016/j.rbce.2018.01.008.

Silva, W. R., Medeiros, T. E., Greboggy, D. L. \& Cardoso, F.L. (2018). Será o trabalho não remunerado o principal determinante para a baixa percepção do status social subjetivo em atletas de futebol de base? Revista iberoamericana de psicología del ejercicio y el deporte. 13(2), 267-271.

Tedesco, J. (2014). Exportacão de pés: Jogadores brasileiros de futsal na Itália e redes transnacionais. Revista de Antropologia Social; 15(1), 57-74.

Zangirolami-Raimundo, J., Echeimberg de Oliveira, J. \& Leone, C. (2018). Tópicos de metodologia de pesquisa: estudos de corte transversal. Journal of Human Growth and Development, 28(3), 11-28.

Zaratim, S. (2012). Aspectos Socioculturais do futsal. Revista Eletrônica De Educação Da Faculdade Araguaia; 2(2), 